NASA Technical Memorandum 106113

$18602 ?$

ICOMP-93-11

CMOTT-93-3

\title{
A Multiple-Scale Turbulence Model for Incompressible Flow
}

B.S. Duncan

Sverdrup Technology, Inc.

Lewis Research Center Group

Cleveland, Ohio

and

W.W. Liou and T.H. Shih

Institute for Computational Mechanics in Propulsion

and

Center for Modeling of Turbulence and Transition

Lewis Research Center

Cleveland, Ohio

Prepared for the

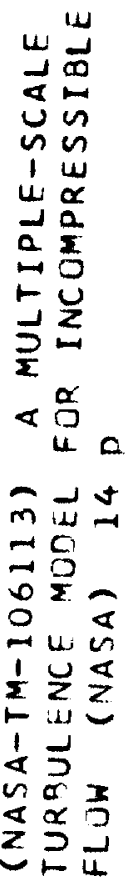

31st Aerospace Sciences Meeting and Exhibit sponsored by the American Institute of Aeronautics and Astronautics Reno, Nevada, January 11-14, 1993

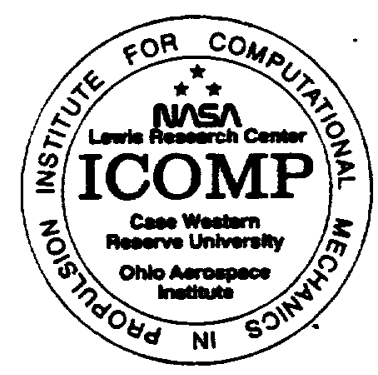


$-$

-

( 


\title{
A MULTIPLE-SCALE TURBULENCE MODEL FOR INCOMPRESSIBLE FLOW
}

\author{
B.S. Duncan \\ Sverdrup Technology, Inc. \\ Lewis Research Center Group \\ Brook Park, Ohio 44142 \\ W.W. Liou and T.H. Shih \\ Institute for Computational Mechanics in Propulsion \\ and Center for Modeling of Turbulence and Transition \\ Lewis Research Center \\ Cleveland, Ohio 44135
}

\section{Abstract}

A multiple-scale eddy viscosity model is described in this paper. This model splits the energy spectrum into a high wave number regime and a low wave number regime. Dividing the energy spectrum into multiple regimes simplistically emulates the cascade of energy through the turbulence spectrum. The constraints on the model coefficients are determined by examining decaying turbulence and homogeneous turbulence. A direct link between the partitioned energies and the energy transfer process is established through the coefficients. This new model has been calibrated and tested for boundary-free turbulent shear flows. Calculations of mean and turbulent properties show good agreement with experimental data for two mixing layers, a plane jet and a round jet.

\section{Introduction}

In turbulent flows, the mean flow performs deformation work which tansfers energy from the mean flow to the largescale turbulent eddies. The turbulent kinetic energy contained within these large eddies is passed to the smaller eddies by vortex stretching. Once the energy has been passed into eddies near the Kolmogorov scale, it is then dissipated by the molecular viscosity. This process can be thought of as a turbulent kinetic energy cascade [1]. In other words, the turbulent kinetic energy is passed through the wave number spectrum as it cascades from large to small eddies.

The multiple-scale turbulence model which is proposed in this study splits the energy spectrum into low and high wave

\footnotetext{
"Member of Center for Modeling of Turbulence and Trancition

tMember of Center for Modeling of Turbulence and Trancition

tTechnical Leader, Center for Modeling of Turbulence and Trancition

${ }^{\circ}$ Copyright (C1992 by the American Inntitute of Aeronautics and Astronautica, Inc. No copyright is asserted in the United State under Title 17, U.S. Code. The U.S. Government has a royalty-free license to exencise all rights under the copyright claimed herein for Governmentel purposes. All other rights are reserved by the copyright owner.
}

number regions. The low wave number region contains the large-acale eddies and the high wave number region consists of the smaller, less energetic eddies. A division of this nature models the cascade of energy from the production region where the energy is initially created by the mean straining work to the disaipation region where this energy is eventually disaipated. This concept is illustrated in Figure 1. Although a simple two-part division of the energy spectrum cannot fully model the cascade of energy, it can simulate the nonequilibrium energy transfer process which is beyond the capability of all single-acale models.

This concept was incorporated into an earlier multiple-time scale turbulence model by Hanjalic, Launder and Schiestel [2] (hereafter HL\&S). Based on the same modeling methodology that was used in the development of the "standard" $k-c$ model [3], HL\&S derived four transport equations to dearibe the turbulent characteristics of the two regions. Consequently, HL\&SS formulated two transport equations for the partitioned turbulent kinetic energies. They also developed transport equations for the rate of energy transfer between the two scales and for the rate of energy transfer to the small-scale eddies. In this model, the coefficients were written as functions of both the ratio of the partitioned energies and the ratio of the spectral energy transfer rates. A term containing the mean vorticity was included in the energy transfer rate equation to account for the increased energy transfer rates in irrotational shear flows. Their results [2] showed fairly good agreement between the model predictions and the experimental data for jets and boundary layers.

Kim and Chen [4] (hereafter $K \& C$ ) developed another multiple-scale model based on the energy partitioning idea introduced by HL\&S. In this model, the transport equations were modified to include an extra source term in both the energy transfer rate equation and the energy dissipation rate equation. In addition, the turbulent velocity scale was characterized by the total turbulent kinetic energy. $\mathrm{K} \& \mathrm{C}$ calibrated the model constants which appear in the energy transfer equations for simple, wall-bounded turbulent flow problems. This model has been used for several boundarylayer flow problems using the appropriate near-wall corrections. 
The model in this study is also based on the energy partitioning concept of HLdzS. In particular, the model coeffcients are dynamically dependent upon the partitioning of the energy spectrum. The variable nature of the coefficients adjusts the model to different flow situations. There is no need for the extra source terms used in K\&C's model or the rotational straining term in the HL\&S model. These model coefficients have been calibrated for homogeneous shear flow and decaying grid turbulence. The present moltiple-acale turbulence model has been teated for boundary-free shear flows. Two mixing layers at different apeed ratios, a planar jet and a round jet have been evaluated. All the computar tions show reasonably good agreement with the data.

\section{Model Equations}

\section{Mean Flow Equations}

For incompressible turbulent flow, the ensembled-averaged equations for continuity of mass and momentum are written as

$$
\frac{\partial U_{i}}{\partial x_{i}}=0
$$

and

$$
\frac{D U_{i}}{D t}=\frac{\partial}{\partial x_{j}}\left(\nu \frac{\partial U_{i}}{\partial x_{j}}-\overline{u_{i} u_{j}}\right)-\frac{1}{\rho} \frac{\partial P}{\partial x_{i}}
$$

where $-\overline{u_{i} u_{j}}$ is the turbulent Reynolds stress tensor. Using the eddy viscosity concept, the Reynolds stress can be related to the mean strain rate and a turbulent eddy viscosity,

$$
-\overline{u_{i} u_{j}}=\nu_{t}\left(\frac{\partial U_{i}}{\partial x_{j}}+\frac{\partial U_{j}}{\partial x_{i}}\right)-\frac{2}{3} k \delta_{i j}
$$

Now, the momentum equation can be written as

$$
\frac{D U_{i}}{D t}=\frac{\partial}{\partial x_{j}}\left(\left(\nu+\nu_{i}\right) \frac{\partial U_{i}}{\partial x_{j}}\right)-\frac{1}{\rho} \frac{\partial P}{\partial x_{i}} .
$$

The turbulent eddy viscosity, $\nu_{t}$, can be characterized by the local turbulent kinetic energy and the local length scale of the energy containing eddies,

$$
\nu_{t} \propto k^{\frac{1}{3}} l \text {. }
$$

The definition used for this length scale is the primary discriminating factor of eddy viscosity turbulence models. For instance, in a $k-\epsilon$ model this length scale is written in terms of the turbulent kinetic energy, $k$, and its dissipation rate, $\epsilon$,

$$
l=\frac{k^{\frac{3}{3}}}{\epsilon} \text {. }
$$

In the present model, the length scale is described by the total turbulent kinetic energy and the rate of spectral energy transfer, i.e. $l=\frac{k l}{l y}$. The turbulent kinetic energies and the rates of energy transfer are determined by modeled transport equations similar to the "standard" $k-c$ equations. These transport equations are described in the following section.

\section{Turbulence Equations}

In this multiploseale turbulence model, the energy spectrum has been aplit into a region where the turbulent kinetic energy has been produced by interaction with the mean flow and a region where the turbulent energy has been transferred from the production region. This division can be graphically represented by Figure 1 . Now, $k_{p}$ is the kinetic energy contained within the production region and $\epsilon_{p}$ is the rate at which energy is paseed from the low wave number range into the high wave number range. At the high wave number end of the spectrum, $k_{t}$ is the kinetic energy contained in the smaller eddies and $\epsilon_{t}$ is taken to be equivalent to the disaipation rate. The modeled transport equations are

$$
\begin{aligned}
& \frac{D k_{p}}{D t}=\frac{\partial}{\partial x_{j}}\left[\left(\nu+\frac{\nu_{t}}{\sigma_{k_{p}}}\right) \frac{\partial k_{p}}{\partial x_{j}}\right]+P_{k_{p}}-\epsilon_{p} \\
& \frac{D \epsilon_{p}}{D t}=\frac{\partial}{\partial x_{j}}\left[\left(\nu+\frac{\nu_{t}}{\sigma_{\epsilon}}\right) \frac{\partial \epsilon_{p}}{\partial x_{j}}\right]+c_{p 1} \frac{\epsilon_{p}}{k_{p}} P_{k_{p}}-c_{p 2} \frac{\epsilon_{p}^{2}}{k_{p}}(8) \\
& \frac{D k_{t}}{D t}=\frac{\partial}{\partial x_{j}}\left[\left(\nu+\frac{\nu_{t}}{\sigma_{k_{1}}}\right) \frac{\partial k_{t}}{\partial x_{j}}\right]+\epsilon_{p}-\epsilon_{t} \\
& \frac{D \epsilon_{t}}{D t}=\frac{\partial}{\partial x_{j}}\left[\left(\nu+\frac{\nu_{t}}{\sigma_{\epsilon_{t}}}\right) \frac{\partial \epsilon_{t}}{\partial x_{j}}\right]+c_{t 1} \frac{\epsilon_{t} \epsilon_{p}}{k_{t}}-c_{t 2} \frac{\epsilon_{t}^{2}}{k_{t}}
\end{aligned}
$$

where,

$$
P_{k p}=\nu_{t}\left(\frac{\partial U_{j}}{\partial x_{i}}+\frac{\partial U_{i}}{\partial x_{j}}\right) \frac{\partial U_{i}}{\partial x_{j}}
$$

Here, $P_{k p}$ is the production of kinetic energy by the large scale eddies. Note that the $\epsilon_{p}$ term serves as a sink in the equation for $k_{p}$ and a source in the $k_{t}$ equation. The source and sink terms in the energy transfer rate equations $\left(\epsilon_{p}\right.$ and $\epsilon_{t}$ ) are related to those in the turbulent kinetic energy equations by their corresponding time scales, i.e. $\frac{k_{p}}{c_{p}}$ and $\frac{k_{p}}{c_{q}}$.

These transport equations, equations (7) through (10), posses several differences from the earlier multiple-scale models. HL\&S include a rotational straining term in the $\epsilon_{p}$ energy tranfer equation (8) to improve their model's performance in axisymmetric flows. Based on dimensional reasoning, $K \& C$ [4] include additional terms in the $\epsilon_{p}$ and $\epsilon_{t}$ equations which are nonlinear in $P_{k_{p}}$ and $\epsilon_{p}$, respectively. The present model is the least complicated as it uses neither 
the rotational straining term nor the nonlinear production terms for energy transfer rates in the model equations.

Accounting for the multiple partitions in the turbulent kinetic energy spectrum and the spectral energy transfer rates, the eddy viscosity in the present model is defined as

$$
\nu_{t}=c_{\mu} \frac{\left(k_{t}+k_{p}\right)^{2}}{\epsilon_{p}}
$$

and $c_{\mu}=0.09$. This is the same relationship for eddy vis cosity used by K\&C. With this formulation, the single scale eddy viscosity model will be recovered when $\epsilon_{p}$ approaches $\epsilon$ in equilibrium turbulence. The $\sigma$ coefficients in equations (7) through (10) are assumed to be the following constants,

$$
\sigma_{k_{p}}=\sigma_{k_{1}}=1.0 \text { and } \sigma_{t_{p}}=\sigma_{e_{t}}=1.3 \text {. }
$$

The other coefficients in equations (7) through (10), namely $c_{p 1}, c_{p 2}, c_{t 1}$ and $c_{t 2}$, are the modeling coefficients discussed in the following section.

\section{Model Coefficients}

The coefficients for this incompressible model have been determined from analyses of homogeneous and decaying turbulence.

\section{Grid Turbulence}

In homogeneous decaying grid turbulence, the turbulent quantities are functions of time only and equations (7) through (10) can be simplified to

$$
\begin{aligned}
\frac{d k_{p}}{d t} & =-\epsilon_{p} \\
\frac{d \epsilon_{p}}{d t} & =-c_{p 2} \frac{\epsilon_{p}^{2}}{k_{p}} \\
\frac{d k_{t}}{d t} & =\epsilon_{p}-\epsilon_{t} \\
\frac{d \epsilon_{t}}{d t} & =c_{t 1} \frac{\epsilon_{t} \epsilon_{p}}{k_{t}}-c_{t 2} \frac{\epsilon_{t}^{2}}{k_{t}} .
\end{aligned}
$$

Most of the experimental evidence suggests that the turbulent kinetic energy decays in time and can be represented by

$$
\frac{k_{p}}{k_{p 0}}=\left(\frac{t}{t_{0}}\right)^{-n}
$$

and

$$
\frac{k_{t}}{k_{t 0}}=\left(\frac{t}{t_{0}}\right)^{-n}
$$

where $n$ is the decay rate and is typically of the order 1.2 . From the above kinetic energy equations, (14) and (16), the energy transfer rates must decay as

$$
\frac{\epsilon_{p}}{\epsilon_{p 0}}=\left(\frac{t}{t_{0}}\right)^{-n-1}
$$

and

$$
\frac{\epsilon_{t}}{\epsilon_{t 0}}=\left(\frac{t}{t_{0}}\right)^{-n-1} .
$$

Therefore, the $c_{p 2}$ coefficient from equation (15) can be related to the decay rate,

$$
c_{p 2}=\frac{n+1}{n} .
$$

Manipulating the simplified transport equations, (14) through (17), and using the relations given by equations (18) through (21) yields a relationship between $c_{12}$ and the other coefficients,

$$
c_{t 2}=\frac{c_{11}+\frac{k_{1}}{k_{p}} c_{p 2}}{\frac{k_{p}}{k_{p}}+1}
$$

\section{Homogeneous Shear Flow}

Guidelines can be established for determining the remaining two constants, $c_{p 1}$ and $c_{t 1}$, by examining the physical behavior of homogeneous shear flows. In this flow situation, the turbulent transport equations reduce to

$$
\begin{aligned}
& \frac{d k_{p}}{d t}=P_{k p}-\epsilon_{p} \\
& \frac{d \epsilon_{p}}{d t}=c_{p 1} \frac{\epsilon_{p}}{k_{p}} P_{k p}-c_{p 2} \frac{\epsilon_{p}^{2}}{k_{p}} \\
& \frac{d k_{t}}{d t}=\epsilon_{p}-\epsilon_{t} \\
& \frac{d \epsilon_{t}}{d t}=c_{t 1} \frac{\epsilon_{t} \epsilon_{p}}{k_{t}}-c_{t 2} \frac{\epsilon_{t}^{2}}{k_{t}} .
\end{aligned}
$$

If we define

$$
\alpha=\frac{P_{k p}}{\epsilon_{t}}
$$

and

$$
\beta=\frac{\epsilon_{p}}{\epsilon_{t}},
$$

then, equation (24) can be combined with (25) to give

$$
\frac{\epsilon_{p}}{k_{p}} \frac{d k_{p}}{d \epsilon_{p}}=\frac{(\alpha-\beta)}{c_{p 1} \alpha-c_{p 2} \beta}
$$

Likewise, equations (26) and (27) can be combined with the following result,

$$
\frac{\epsilon_{t}}{k_{t}} \frac{d k_{t}}{d \epsilon_{t}}=\frac{\beta-1}{c_{t 1} \beta-c_{t 2}}
$$


Assuming that $\beta$ and the percentages of the kinetic energy contained in $k_{p}$ and $k_{t}$ remain nearly constant, then

$$
\frac{\epsilon_{p}}{k_{p}} \frac{d k_{p}}{d \epsilon_{p}}=\frac{\epsilon_{t}}{k} \frac{d k}{d \epsilon_{t}}=\frac{(\alpha-\beta)}{c_{p 1} \alpha-c_{p 2} \beta} .
$$

and

$$
\frac{\epsilon_{t}}{k_{t}} \frac{d k_{t}}{d \epsilon_{t}}=\frac{\epsilon_{t}}{k} \frac{d k}{d \epsilon_{t}}=\frac{\beta-1}{c_{t 1} \beta-c_{t 2}} .
$$

From the experiment of [5], the ratio $\frac{c_{k}}{k} \frac{d k}{d d_{1}}$ has been deduced to be 1.065 . In a similar experiment, Tavoularis and Corrisin [6] found this ratio to be between 0.82 and 0.94 . Clearly, this ratio is on the order of one. For simplicity, this term, $\frac{\&}{b} \frac{d k}{d e_{1}}$ , has been ascumed to be unity. Therefore, the following expressions for $c_{p 1}$ and $c_{11}$ can be found from equations (32) and (33),

$$
\begin{gathered}
c_{p 1}=\left(1-\frac{\beta}{\alpha}\right)+\frac{\beta}{\alpha} c_{p 2} \\
c_{t 1}=\frac{\beta-1}{\beta}+\frac{c_{t 2}}{\beta} .
\end{gathered}
$$

Equation (23) is now,

$$
c_{t 2}=\frac{\beta-1+c_{p 2} \beta \frac{k_{p}}{k_{p}}}{\beta+\beta \frac{k_{1}}{k_{p}}-1}
$$

and, $c_{p 2}$ is defined in equation (22). The coefficients, $c_{p 1}, c_{t 1}$ and $c_{t_{2}}$ are functions of $\frac{P_{t_{2}}}{c_{t}}, \frac{c_{2}}{c_{1}}$ and $\frac{k_{1}}{k_{7}}$. In the present model, the ratios deacribed in equations (28) and (29) are assumed to be the following constants,

$$
\alpha=\frac{P_{k p}}{\epsilon_{t}}=2.2
$$

and

$$
\beta=\frac{\epsilon_{p}}{\epsilon_{t}}=1.05 \text {. }
$$

These constants have been calibrated considering that experimental measurments of homogeneous shear flow suggest that the ratio $\frac{\alpha}{\beta}$ should be near two. These coefficients are summarized in Table 1.

Notice that the value of $\frac{k_{1}}{k_{p}}$ is allowed to vary as the ratio of the turbulent kinetic energy in the small scales to the energy contained in the large scales changes. Since most of the energy is contained in the large scales, this ratio should remain less than one [1]. This coefficient adaptability allows the model to adjust to different flow situstions and is a unique characteristic of multiple-scale eddy viscosity models.

\section{Results and Discussions}

The present multiple-scale model has been tested for two planar mixing layers and two jet flows. As part of these teots, the model has been compared to experimental data and to the multiplo-ecale models of Hanjalic, Launder and Schiestel [2] (HL\&S) and Kim and Chen [4] (K\&C) and to the "standard" $k-\epsilon$ model. In all cases, the parabolic solution technique is started with an initial plane and the flow field evolves as the computations march in the arial direction. The solutions are checked to insure that they maintain a self-perserving profile. The results of these calculations are presented in Figures 2 through 18 and in Table 2.

\subsection{Planar Mixing Layers}

For the planar miring layers, the flow is ascumed to have a thin shear layer profile at the interface between the still air and the jet. Zero gradient boundary conditions for the turbulence quantities are applied at the edges of the flow field and an equally spaced grid is used.

Speed ratio $=0.0$

In Figure 2, the three multiple-scale models and the "standard" $k-$ model are compared to the experimental dats of Wyganati and Fielder[7] and Patel [8]. At the high speed edge, none of the four models predicts the diffunive characteriatic indicated by the data. Away from this area, however, the present model, HL\&S's model and the "standard" $k-c$ model all are very cloee to Patel's data. The data due to Wygnanaki and Fielder is considerably more diffusive than either Patel's dats or the computations. The spreading rates predicted by the four eddy viscosity models and the spreading rate measured by Patel are listed in Table 2. For miring layers, the spreading rate is defined as $\frac{d\left(x . \frac{-y}{d x} \cdot\right)}{d x}$. The spreading rate predictions by the present model and the "standard" $k-\epsilon$ model are closest to the data. K\&z's model significantly underestimates the growth rate of the mixing layer.

The tendency of K\&C's model to under-predict the growth of the turbulent mixing region is further seen in Figures 3 and 4. The shapes of the kinetic energy curves and the shear stres distributions are correct, but the peak levels are well below the data. The "standard" $k-\epsilon$ model, HL\&S's model and the present model predict turbulent hinetic energy levels slightly below the dats but they correctly predict the peak levels for shear stress. Apparently, the computational shear layers tend to shift further towards the low speed side of the flow than the experimentally measured shear layers.

In Figure 5, the ratios of the partitioned hinetic energies and the energy transfer rates are shown. Notice that the ratio, $\frac{i_{1}}{g_{p}}$, is one through the mixing layer where the turbulence spectrum is in equilibrium. The fact that $\frac{k_{1}}{k_{y}}$ remains much leas than one indicates that most of the energy is contained 
by the large seale eddies as stated by Tennekes and Lumley [1].

\section{Speed ratio $=0.3$}

The next case considered is a mixing layer with a speed ratio of 0.3. In Figure 6, the mean velocity profiles predicted by all four models are in very good with the experimental data measured by Spencer et. al. [9]. The present model and the "standard" $b-$ model predict an almoet identical velocity profile. HL\&S's and K\&C's models under-predict the growth of the mixing region which is indicated by the narrower mean velocity profile and also by the lower spreading rates listed in Table 2.

Looking at turbulent quantities, the "standard" $k-\epsilon$ and the present $k_{p}-\varepsilon_{p}-k_{t}-t_{t}$ model predict the kinetic energy distribution very well as can be seen in Figure 7. Although the models due to HL\&S and K\&C yield good mean velocity predictions, they both under-predict the peak turbulent kinetic energy level. The present model and the "gtandard" $k-\epsilon$ model yield very good predictions of the turbulent $k i-$ netic energy profile. All the eddy viscosity models in this study under-predict the peak shear stress shown in Figure 8. Here, the "standard" $k-\epsilon$ and the present model do the best job of predicting the shear stress profile however, they are both slightly low in their predictions of the peak value. Again, the other two models are quite low in their predictions of the peak turbulent shear stress.

\section{Grid Resolution Analysis}

Since the mixing layer with a speed ratio of 0.3 gives the thinnest shear layer and the slowest growth rate, this case would be the most dependent upon grid resolution. For all the calculations discussed thus far there were 65 points across the flow field. Figures 9 and 10 compare the solutions obtained with 65 points to the same calculation with 101 points across the domain. As these Figures indicate, the results are essentially unchanged as the grid is refined.

\subsection{Planar Jet}

For the jet flow simulations, the initial plane is split with a uniform velocity and kinetic energy profile comprising approximately half of the domain and quiescent air comprising the other half. Zero gradient boundary conditions are applied at the centerline of the jet and the grid has been clustered towards the centerline to improve the accuracy of this boundary condition.

As can be seen in Figure 11, the mean velocity profiles predicted by the present model, K\&C's model and the "stan- dard" $k-\epsilon$ model lie within the scatter of the experimental data of [10], [11] and [12]. The present multiple-scale model is particularly close to the dats of Heskestad [12]. Again, the HL\&S model under-predicts the turbulent growth rate which can be inferred by the narrow mean velocity profile and the predicted spreading rate. (During this atudy, the HL\&S model has been found difficult to use. The model appears to be sensitive to initial conditions, marching step sire, etc. This is especially true for the planar and ariogmmetric jets flows.) With the exception of HL\&LS's model, there is very little disagreement between the predicted spreading rates and experimental spreading rate given in Table 2. For jet flows, the opreading rate is defined as the rate of change of the half velocity point, i.e. $\frac{d y}{d s}$. The predicted spreading rates range from 0.103 by $\bar{K} \& C$ 's model to 0.114 by the "standard" $k-t$ model. All these spreading rateo are in reasonably good agreement with the experimentally determined rates.

There is considerably more disagreement between the models for the predicted turbulent kinetic energy levelo, shown in Figure 12. HL\&S's model under-predicts the kinetic energy profile which is not surprising given the low predicted spreading rate. Both the present model and K\&C's model predict relatively flat profiles which match the data of Heskeatad [12]. The "standard" $k-c$ model is in very good agreement with the experimental dats, being expecially close to Bradbury's measurements.

Both multiple-scale models and the "standard" $k-\epsilon$ model correctly predict the peak shear stress shown in Figure 13 although they tend to exaggerate the width of the jet. The model by HL\&S under-predicts the peak shear stress, again due to the under-prediction of the growth rate for the jet.

Figure 14 shows the ratios of $\frac{k_{2}}{k_{1}}$ and $\frac{c_{1}}{c_{3}}$ through the jet for the present multiple-acale model. Notice that these ration are nearly constant and are therefore consistent with the assumption made in deriving the modeling constants. The ratio, $\frac{e s}{c_{2}}$, is near one, except near the centerline of the jet, indicating that the energy spectrum is in equilibrium. On the other hand, the ratios of kinetic energy are much smaller, indicating that most of the energy is in the larger scales.

\subsection{Round Jet}

Using the "standard" $k-t$ turbulence model often gives poor predictions for the spreading rate and flow properties of a round jet. There are several methods to adjust the "standard" $k-c$ model, e.g. by changing the coefficient in the dissipation equation or by adding a vortex stretching term [13]. The "standard" $k-\epsilon$ model predictions given in Figures 15 through 17 have applied no correction methods. As 
previously mentioned, a rotational straining term is used by HL\&S in their multiple-acale model to decrease the energy transfer rate from the production region in rotational flows. Neither the present model nor K\&C's model uses a three-dimensional correction term; yet, as shown in Figure 15 , they give the beat match to the experimental data of Rodi [14] and Wygnanaki \& Fielder [15] for mean velocity.

The turbulent kinetic energy is over-predicted by the "standard" $k-c$ model and under-predicted by HL\&S's model as shown in Figure 16. Closer to the data, but still not giving good predictions, are the present model and $\mathrm{K} \& \mathrm{C}$ 's model. Both models predict the correct centerline turbulent binetic energy, however, the outer edges of the jet are over-predicted. None of the models in this report does a good job of predieting the turbulent binetic energy for an axisymmetric jet.

Figure 17 shows that there is also 8 wide spread in the shear stress predictions. Without adjusting the modeling coefficients or adding correction terms the "standard" $k-c$ model predicts shear strese levels almost double those given by the data. Both the present model and K\&C's model are relatively cloee to the data compared to the "standard" $k-$ $\epsilon$ model. Although, both models over-predict the peak level by approrimately 25 percent. HL\&S's model predicts the narrowest shear stress profile in Figure 17 compared to the other models. Not surprisingly then, the HL\&S model gives the best prediction for the spreading rate. The other two multiple-scale models, i.e. the present model and K\&C's model, predict spreading rates which are 17 and 24 percent too large, respectively, in comparison to the data.

Looking at the ration of $\frac{k_{1}}{k_{y}}$ and $\frac{c_{1}}{c_{p}}$, shown in Figure 9, the flow field is not in equilibrium and the energy transfer to the dissipative acales increases near the centerline of the jet. The "standard" $k \rightarrow$ model has no means to account for the increase in energy transfer rate near the centerline of the jet and consequently over-predicts the turbulent kinetic energy and the spreading rate.

\section{Conclusions}

A multiple-scale eddy viscosity turbulence model with solution dependent coefficients has been developed. This new model splits the energy spectrum in two regions thereby introducing two characteristic length scales. One length scale is associated with the turbulent large-seale eddies which are responsible for generating turbulent kinetic energy by interactions with the mean flow while the other length scale corresponds to the smaller and lese energetic eddies. This partitioning of energy between the large scales and the amall scales crudely emulates the cascade of energy from its production by the mean flow to its eventual dissipation by the molecular viscosity. This modeling concept can simulate the nonequilibrium energy transfer process.

In this paper, the multiple-scale turbulence model has been calibrated using decaying grid turbulence and homogeneous shear turbulence. This model has been tested against two mixing layere at different speed ratios, a planar jet and an axisymmetric jet. For all cases, the mean flow and turbulent propertica have compared reasonably well with the experimental data.

\section{References}

[1] H. Tennekes and J. L. Lumley, A First Course in Turbulence. The MIT Press, 1972.

[2] K. Hanjalic, B. E. Launder, and R. Schieatel, "Multiple-time-scale concepts in modeling turbulent flows," in Turbulent Shear Flows, (New York), Springer-Verlag, 1980.

[3] W. P. Jones and B. E. Launder, "The prediction of laminarization with a two-equation model of turbulence," J. of Heat Mass Transport, vol. 15, pp. pp. 301-314, 1972.

[4] S. W. Kim and C. P. Chen, "A multiplo-time-scale turbulence model based on variable partioning of turbulent kinetic energy spectrum." NASA CR-179222, 1987.

[5] V. G. Harris, J. A. H. Graham, and S. Corrsin, "Further experiments in nearly homogeneous turbulent shear flow," Joxrnal of Fluid Mechanics, vol. 81, 1977.

[6] S. Tavoularis and S. Corrsin, "Experiments in nearly homogeneous turbulent shear flow with a uniform mean temperature gradient. part 1," Journal of Fluid Mechanics, vol. 104, 1981.

[7] I. Wygnanski and H. E. Fiedler, "The two-dimensional mixing region," Journal of Fluid Mechanics, vol. 41, pp. 327-361, 1970.

[8] R. Patel, "An experimental study of a plane mixing layer," AIAA Journal, vol. 11, no. 1, 1973.

[9] B. W. Spencer and B. G. Jones, "Statixical investigation of pressure and velocity fields in the turbulent twostream mixing layer." ALAA paper 71-0613, 1971. 4th Fluids and Plasms Dynamics Conference, June 21-23, 1991, Palo Alto, California.

[10] E. Gutmark and I. Wygnansti, "The planar turbulent jet," Journal of Fluid Mechanics, vol. 73, pp. 465-495, 1976. 
[11] L. J. S. Bradbury, "The stucture of the self-preserving jet," Journal of Fluid Mechanics, vol. 23, pp. 31-64, 1965.

[12] G. Heskestad, "Hot-wire measurements in a plane turbulent jet," Josmal of Fluid Mechanics, 1965.

[13] S. B. Pope, "An explanation of the turbulent roundjet/plane-jet anomaly," AIAA Journal, vol. 16, March 1978.
[14] W. Rodi, "A new method of analyzing hot-wire rignals in highly turbulent flow and its evaluation in round jet." Disa Information No. 17, 1975.

[15] I. Wygnanski and H. E. Fiedler, "Some measurements in the self-preserving jet," Journal of Fluid Mechanics, vol. 38, pp. 577-612.

\begin{tabular}{|l||l||l|l|}
\hline$c_{\mu}=0.09$ & $n=1.2$ & $\alpha=2.2$ & $\beta=1.05$ \\
$c_{p 1}=\left(1-\frac{\beta}{\alpha}\right)+\frac{\rho}{\alpha} c_{p 2}$ & $c_{p 2}=\frac{n+1}{n}$ & $c_{t 1}=\frac{\beta-1}{\beta}+\frac{c_{t 2}}{\beta}$ & $c_{t 2}=\frac{\beta-1+c_{p 2} \rho \frac{k_{1}}{k_{2}}}{\beta+\beta \frac{k_{s}}{\gamma}-1}$ \\
$\sigma_{k,}=1.0$ & $\sigma_{k_{1}}=1.0$ & $\sigma_{\epsilon_{2}}=1.3$ & $\sigma_{t_{t}}=1.3$ \\
\hline
\end{tabular}

Table 1: Multiple-Scale Turbulence Model Coefficients

\begin{tabular}{|l||l|l|l|l|l|}
\hline & Experiment & $\begin{array}{l}\mathrm{HL} \& \mathrm{~S} \\
k_{\mathrm{p}}-\epsilon_{\mathrm{p}}-k_{t}-\epsilon_{t}\end{array}$ & $\begin{array}{l}\mathrm{K} \& \mathrm{C} \\
k_{\mathrm{p}}-\epsilon_{p}-k_{t}-\epsilon_{t}\end{array}$ & $\begin{array}{l}\text { present } \\
k_{p}-\epsilon_{p}-k_{t}-\epsilon_{t}\end{array}$ & $\begin{array}{l}\text { standard } \\
k-\epsilon\end{array}$ \\
\hline Planar Jet & $0.11-0.12$ & 0.076 & 0.103 & 0.104 & 0.114 \\
Round Jet & $0.085-0.095$ & 0.106 & 0.118 & 0.111 & 0.126 \\
Planar Mixing Layer, $\mathrm{r}=0.0$ & 0.179 & 0.146 & 0.126 & 0.152 & 0.159 \\
Planar Mixing Layer, $\mathrm{r}=0.3$ & 0.052 & 0.061 & 0.064 & 0.078 & 0.082 \\
\hline
\end{tabular}

Table 2: Spreading Rate Comparisons for Free Shear Flow 


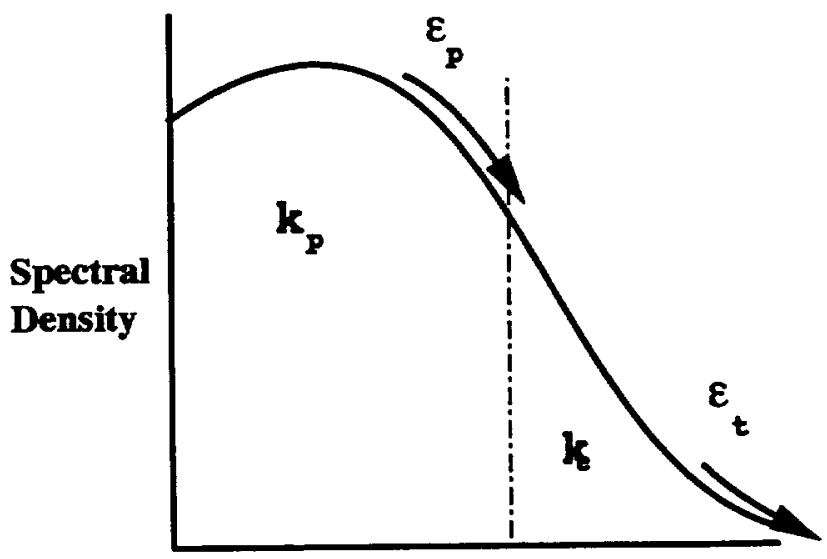

Wave Number

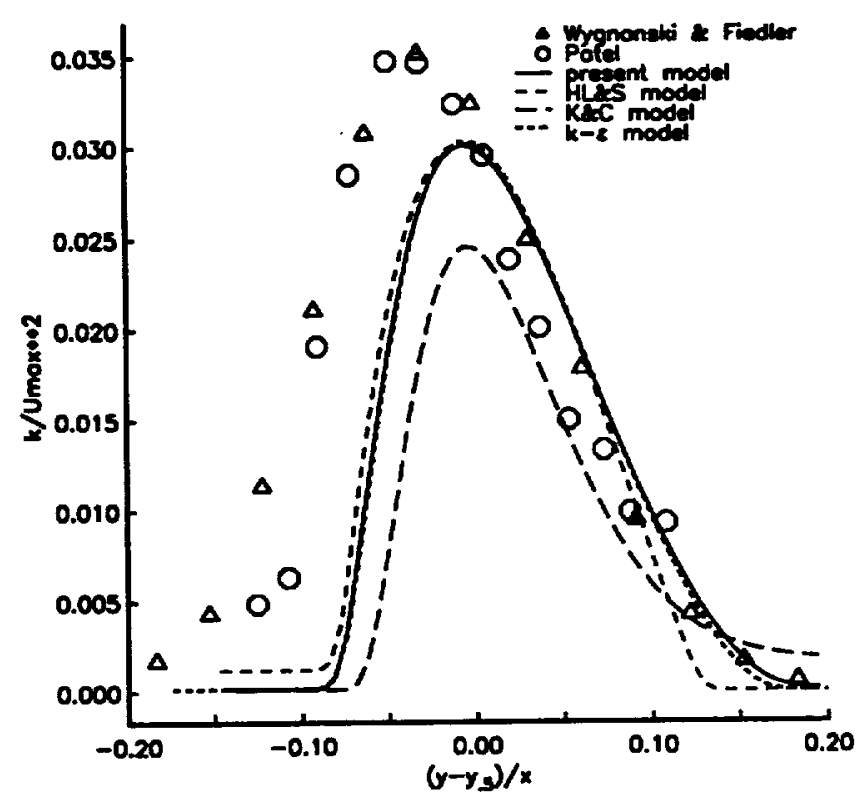

Figure 3: Turbulent kinetic energy profile for a planar mixing layer with a speed ratio of 0 . $U_{\text {mex }}$ : maximum velocity.

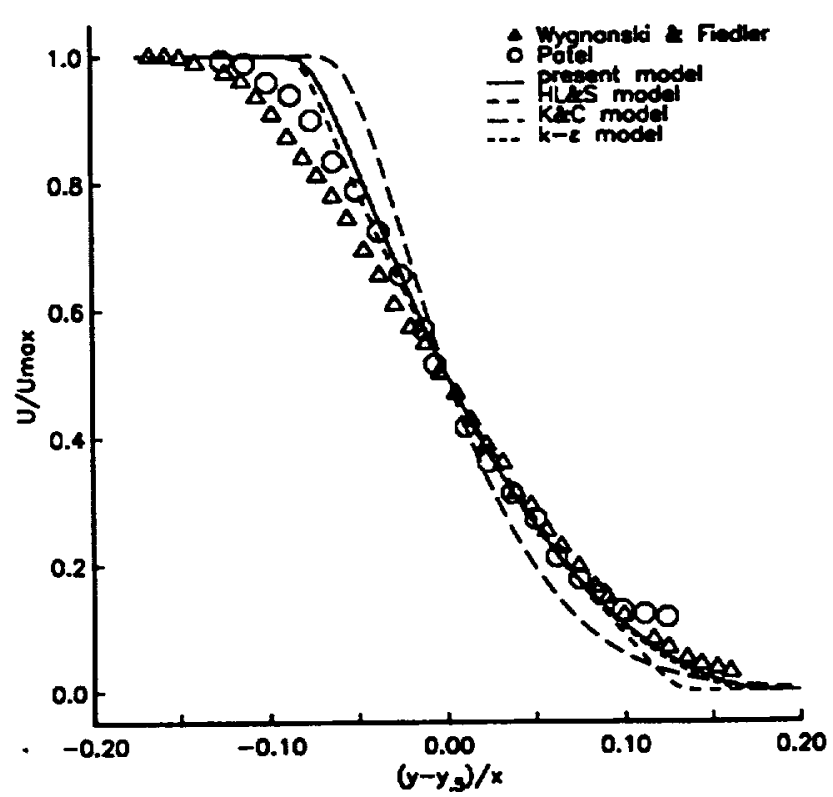

Figure 2: Mean velocity profile for a planar mixing layer with a speed ratio of 0 . $U_{\max }$ : maximum velocity.

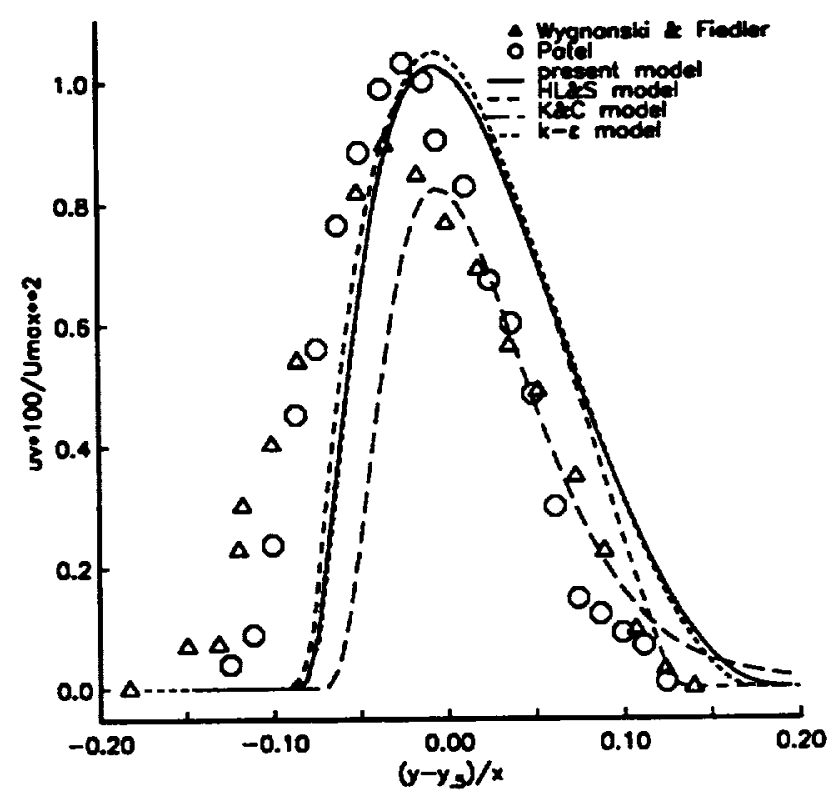

Figure 4: Shear stress profile for a planar mixing layer with a speed ratio of 0 . $U_{\text {max }}$ : maximum velocity. 


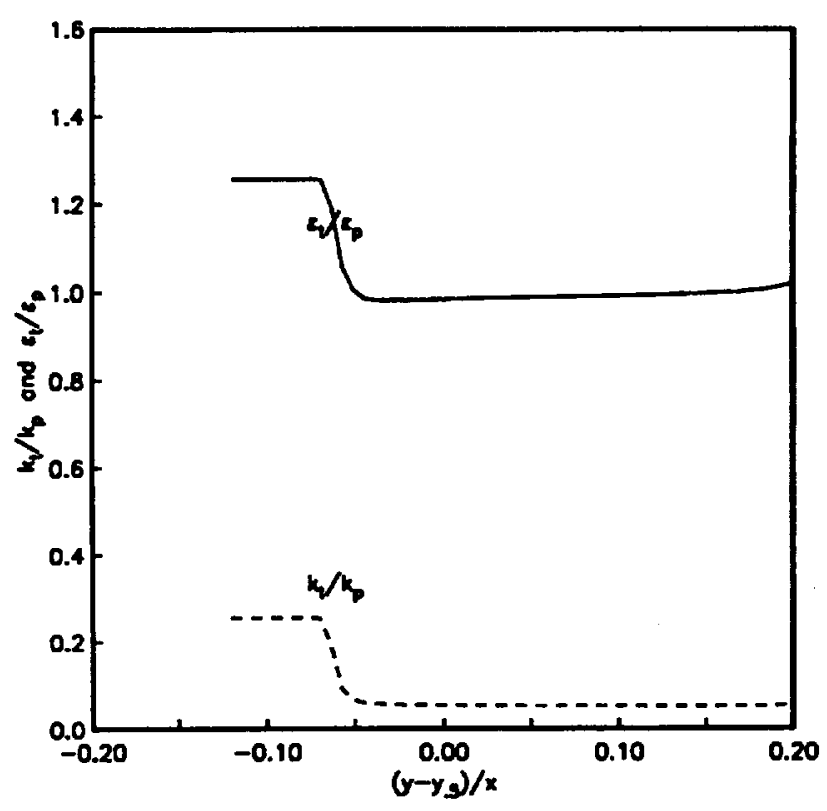

Figure 5: Energy transfer rate and kinetic energy ratios for a plane mixing layer with a speed ratio of 0 .

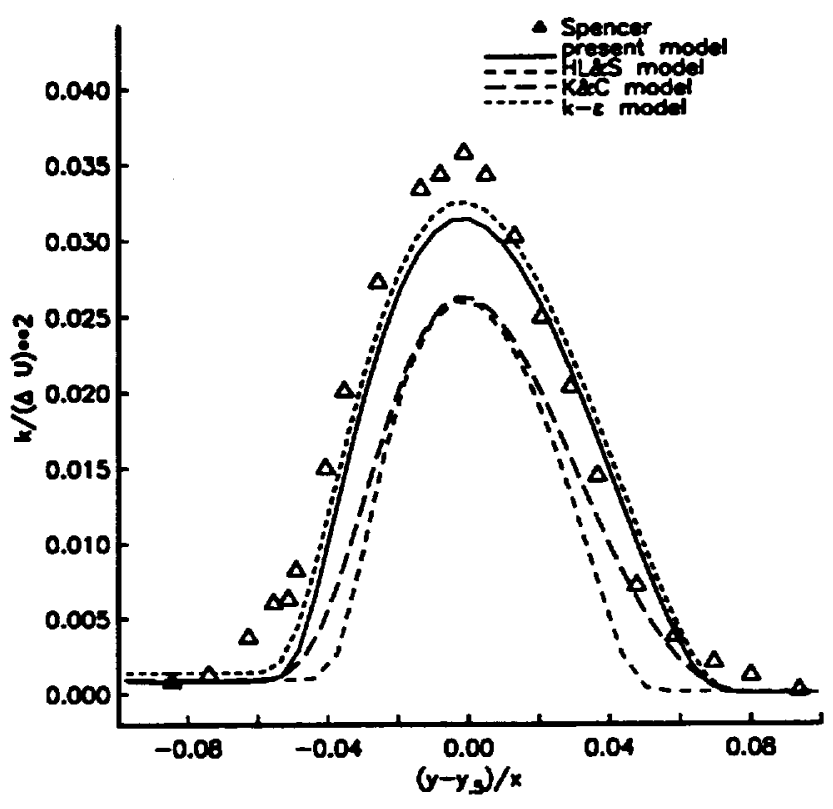

Figure 7: Turbulent kinetic energy profile for a planar mixing layer with a speed ratio of .3. $\Delta U=U_{\max }-U_{\min }$.

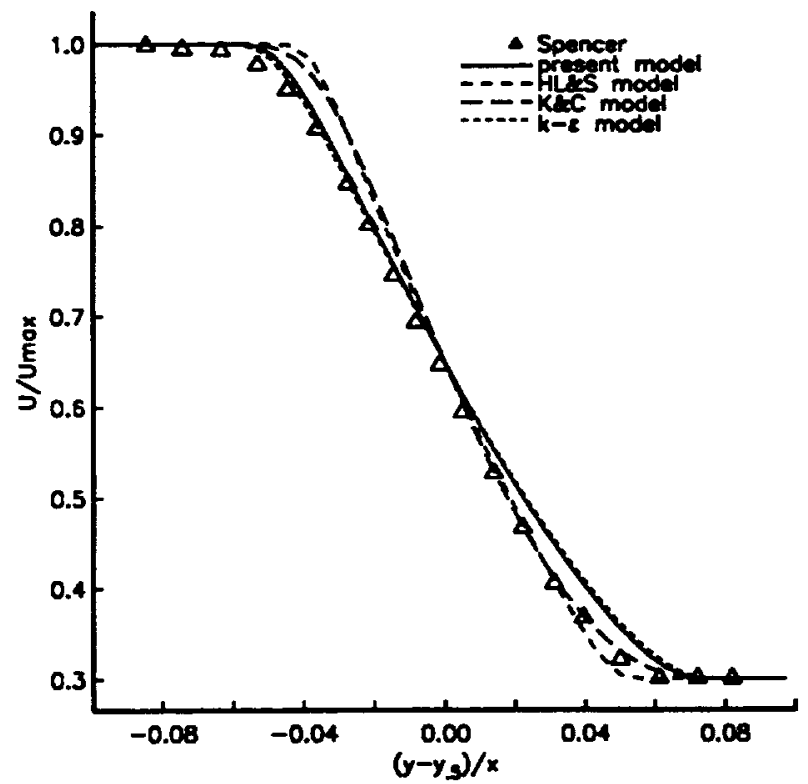

Figure 6: Mean velocity profile for a planar mixing layer with a speed ratio of .3 . $U_{\text {max }}$ : maximum velocity.

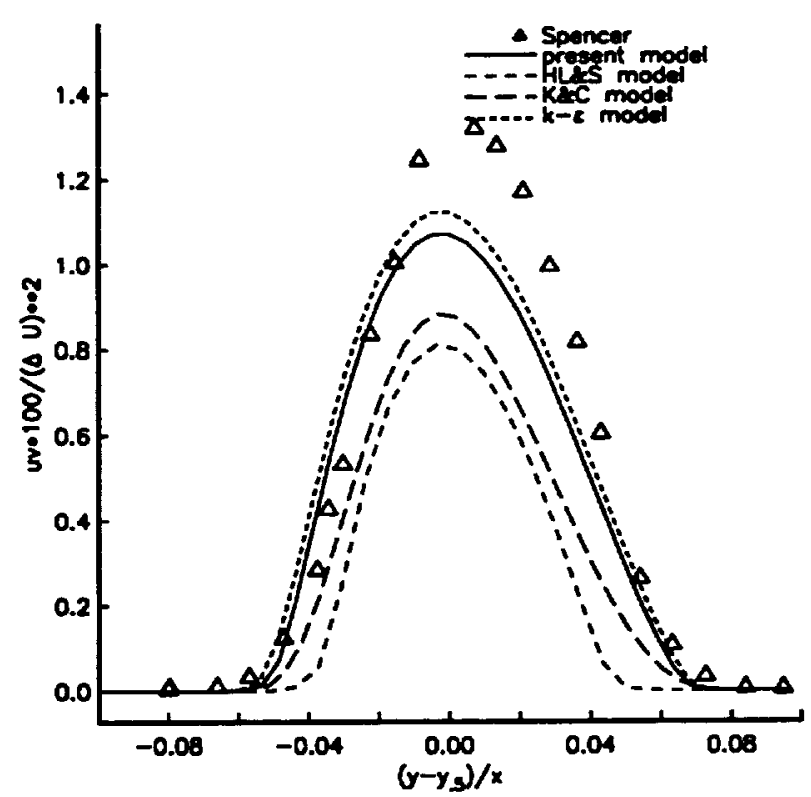

Figure 8: Shear stress profile for a planar mixing layer with a speed ratio of .3. $\Delta U=U_{\max }-U_{\min }$. 


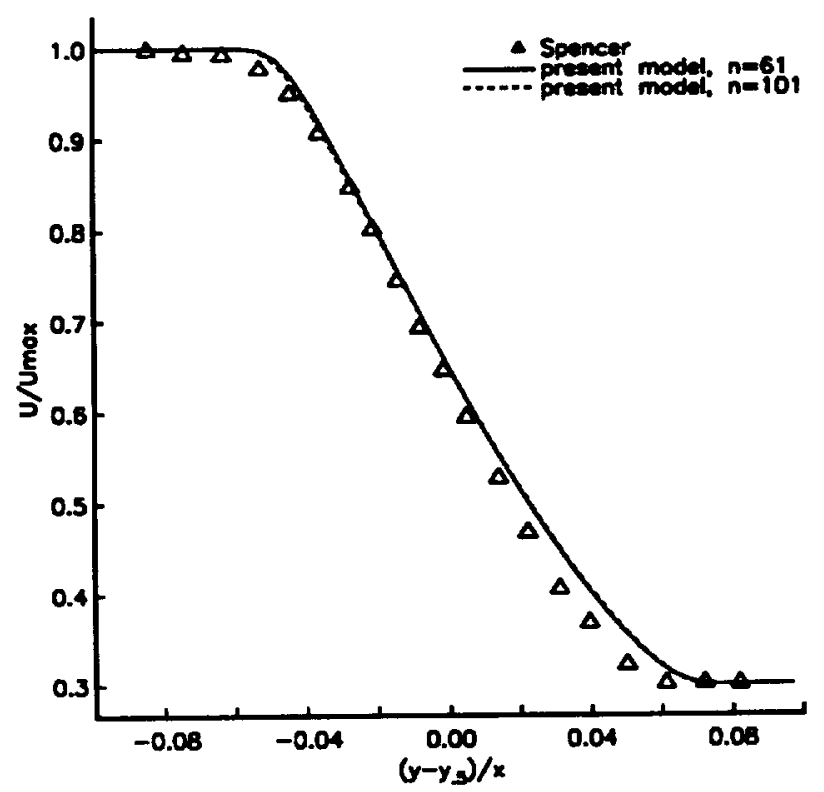

Figure 9: Grid resolution analysis of the mean velocity profile for a planar mixing layer with a speed ratio of .3. $U_{\text {max }}$ : maximum velocity.

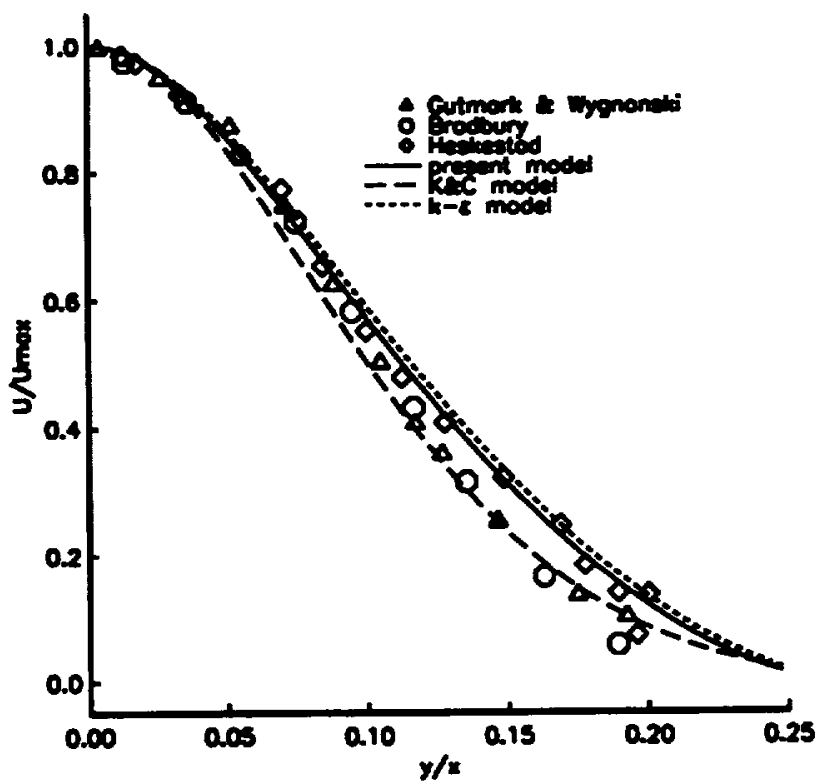

Figure 11: Mean velocity profile for a turbulent, planar jet. $U_{\text {max }}$ : centerline velocity.

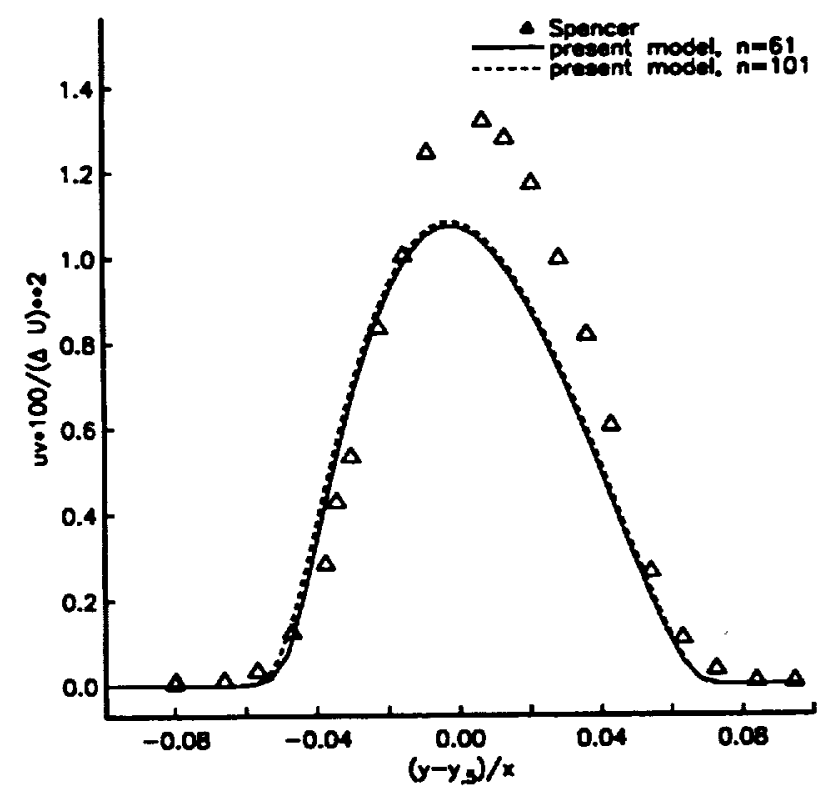

Figure 10: Grid resolution analysis of the shear stress profile for a planar mixing layer with a speed ratio of .3. $\Delta U=$ $U_{\max }-U_{\min }$.

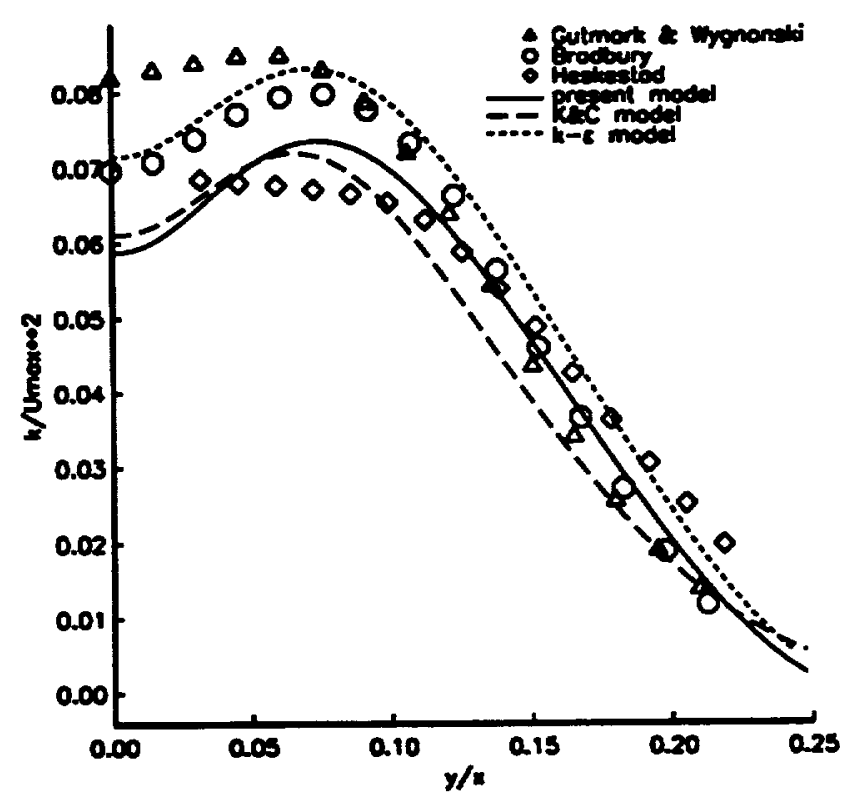

Figure 12: Turbulent kinetic energy profile for a planar jet. $U_{\text {max }}$ : centerline velocity. 


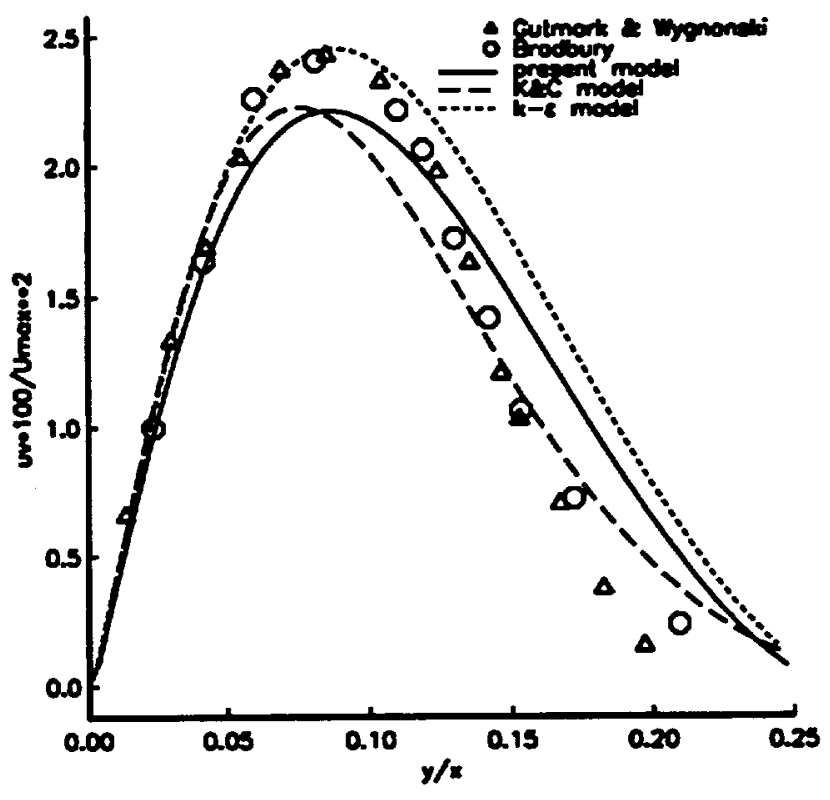

Figure 13: Shear stress profile for a planar jet. $U_{\text {max }}$ : centerline velocity.

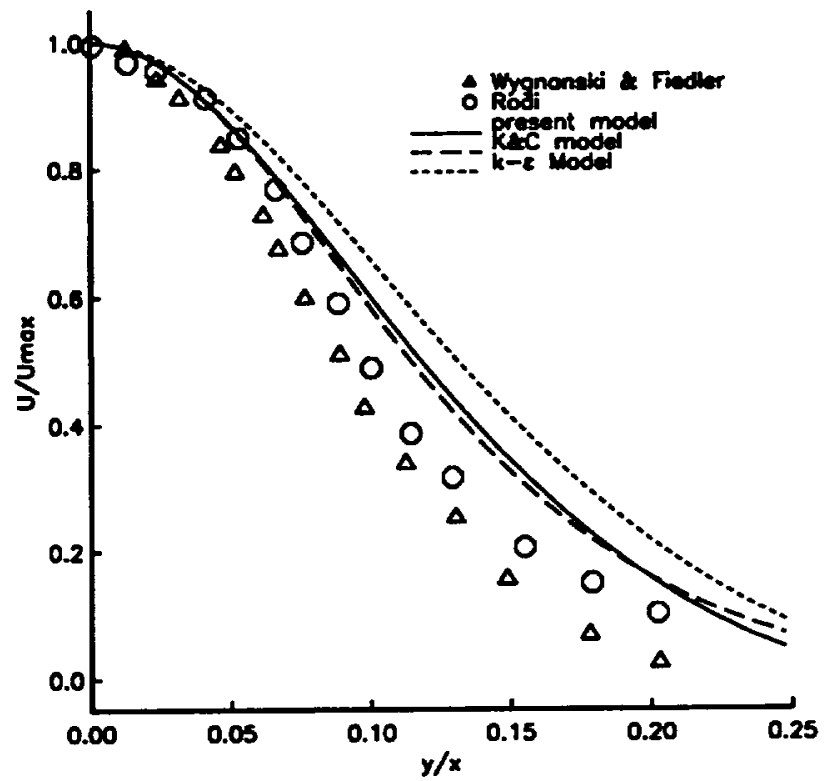

Figure 15: Mean velocity profile for a round jet. $U_{\max }$ : centerline velocity.

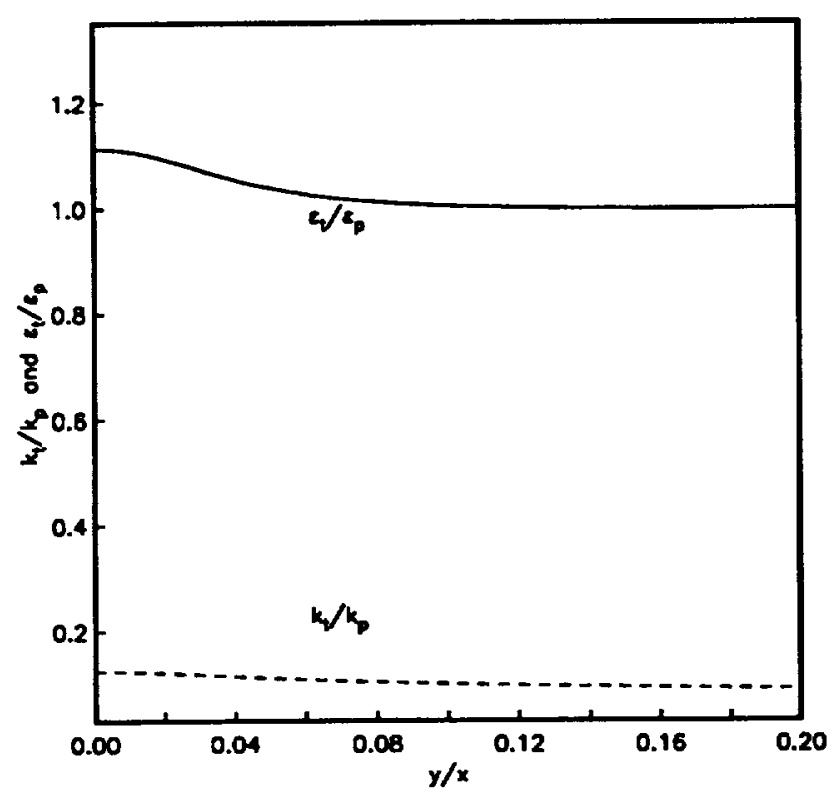

Figure 14: Dissipation and kinetic energy ratios for a planar jet

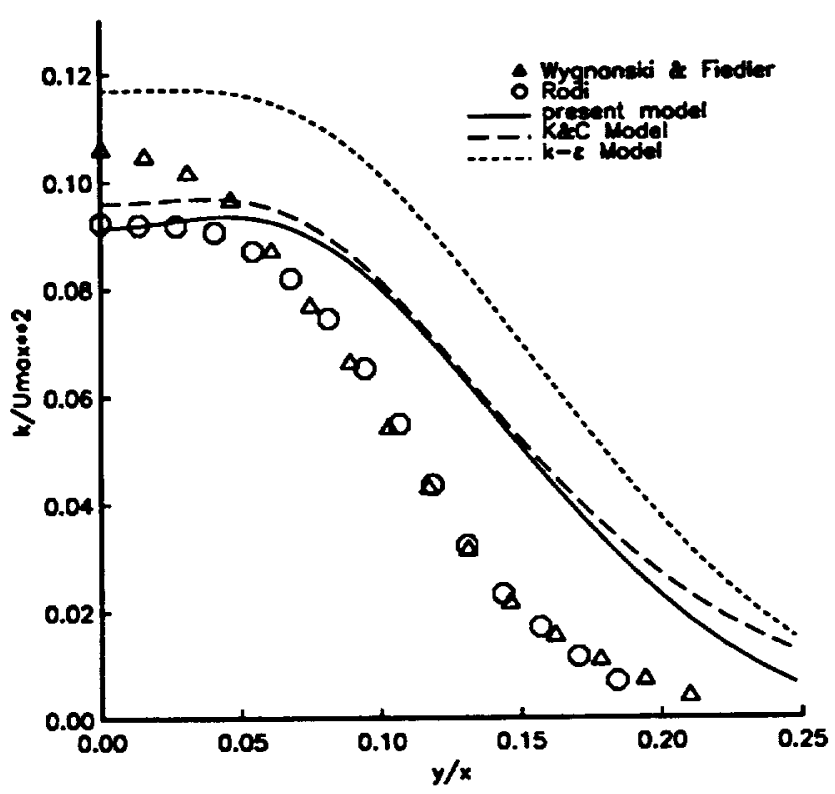

Figure 16: Turbulent kinetic energy profile for a round jet. $U_{\text {max }}$ : centerline velocity. 


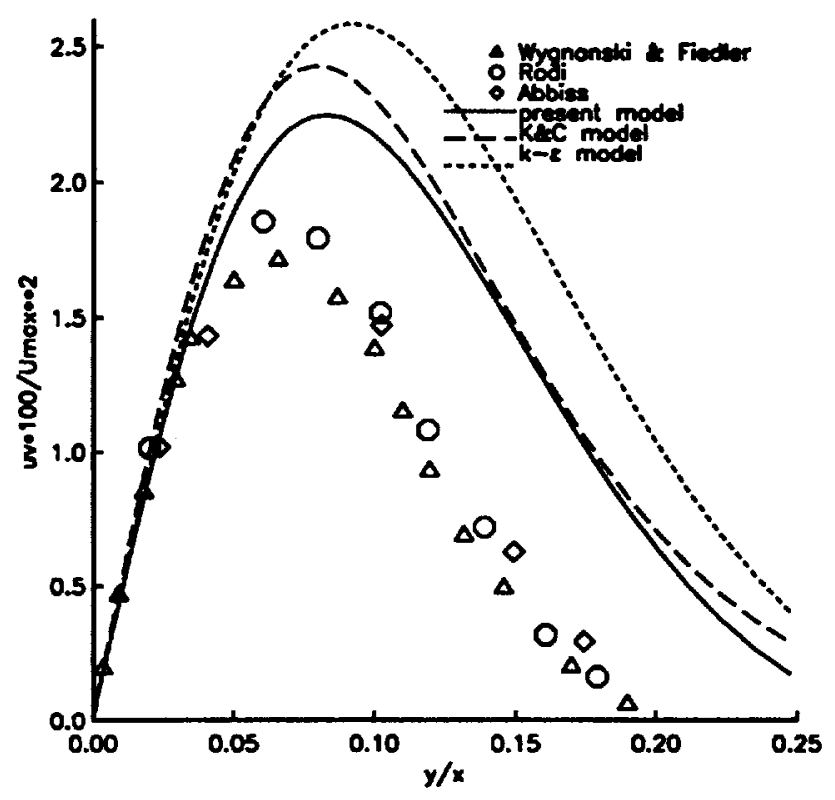

Figure 17: Shear stress profile for a round jet. $U_{\max }$ : centerline velocity.

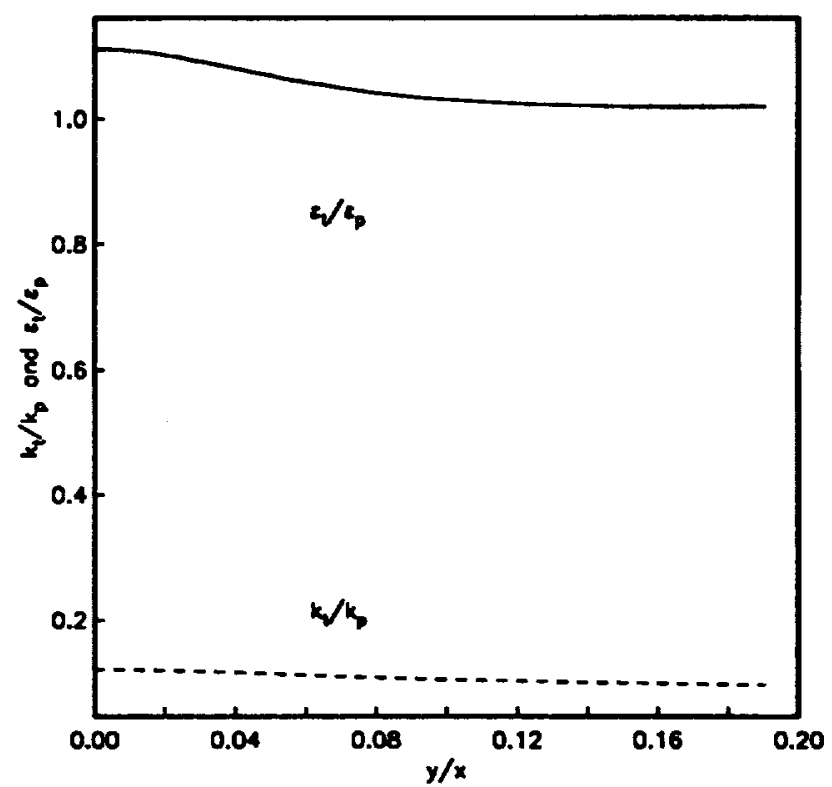

Figure 18: Dissipation and kinetic energy ratios for a round jet. 



\section{REPORT DOCUMENTATION PAGE}

Public reporting burden for this collection of information is estimated to average 1 hour per response, including the time for reviewing instructions, searching existing data sources. gathering and maintaining the data needed, and completing and reviewing the collection of information. Send comments regarding this burden estimate of any other aspect of this collection of intormation, including suggestions for reducing this burden, to Washington Headquarters Services, Directorate for intormation Operations and Reports, 1215 Jeffer
Davis Highway, Suine 1204, Arlington, VA 22202-4302, and to the Office of Management and Budget, Paperwork Reduction Project (0704-0188), Washington. DC 20503.

\begin{tabular}{|l|l|l}
\hline 1. AGEMCY USE ONLY (Leave blank) & $\begin{array}{c}\text { 2 REPORT DATE } \\
\text { January } 1993\end{array}$ & $\begin{array}{r}\text { 3. REPORT TYPE AND DATES COVERED } \\
\text { Technical Memorandum }\end{array}$
\end{tabular}

4. TILE AND SUBTIRE

A Multiple-Scale Turbulence Model for Incompressible Flow

6. AUTHOR(S)

WU-505-62-40

B.S. Duncan, W.W. Liou, and T. H. Shih

7. PERforming organization mame(S) AND ADDRESS(ES)

8. PERForMING ORGANIZATION REPORT NUMBER

National Aeronautics and Space Administration

Lewis Research Center

E-7765

Cleveland, Ohio 44135-3191

9. SPONSORING/MONITORING AGENCY NAME(S) AND ADDRESS(ES)

10. SPONSORING/MONTORING AGENCY REPORT NUMBER

National Aeronautics and Space Administration

Washington, D.C. 20546-0001

NASA TM-106113

ICOMP-93-11

CMOTT-93-3

\section{SUPPLEMENTARY NOTES}

Prepared for the 31st Aerospace Sciences Meeting and Exhibit sponsored by the American lnstitute of Aeronautics and Astronautics, Reno, Nevada, January $11-14,1993$. B.S. Duncan, Sverdrup Technology, Inc., 2001 Aerospace Parkway, Brook Park, Ohio 44142; and W.W. Liou and T.H. Shih, Institute for Computational Mechanics in Propulsion; and Center for Modeling of Turbulence and Transition, Lewis Research Center, Cleveland, Ohio (work funded under NASA Cooperative Agreement NCC3-233). ICOMP Program Director, Louis A. Povinelli, (216) 433-5818.

12a. DISTRIBUTIONAVALABILITY STATEMENT 12b. DISTRIBUTION CODE

Unclassified - Unlimited

Subject Category 34

13. ABSTRACT (Maximum 200 mords)

A multiple-scale eddy viscosity model is described in this paper. This model splits the energy spectrum into a high wave number regime and a low wave number regime. Dividing the energy spectrum into multiple regimes simplistically emulates the cascade of energy through the turbulence spectrum. The constraints on the model coefficients are determined by examining decaying turbulence and homogeneous turbulence. A direct link between the partitioned energies and the energy transfer process is established through the coefficients. This new model has been calibrated and tested for boundary-free turbulent shear flows. Calculations of mean and turbulent properties show good agreement with experimental data for two mixing layers, a plane jet and a round jet.

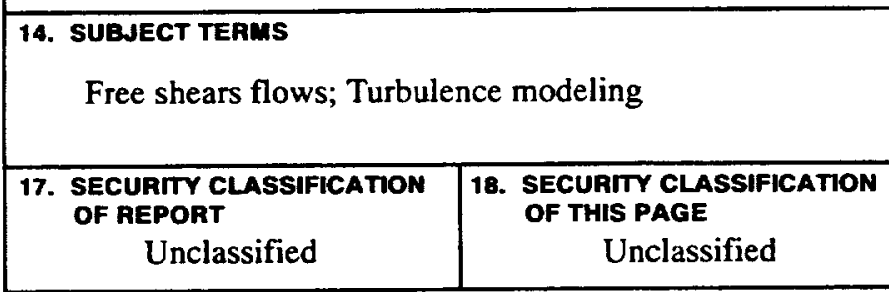

\begin{tabular}{|c|c|}
\hline & $\begin{array}{l}\text { 15. NUMBER OF PAGES } \\
14\end{array}$ \\
\hline & $\begin{array}{r}\text { 16. PRICE CODE } \\
\text { A03 }\end{array}$ \\
\hline $\begin{array}{l}\text { 19. SECURTY CLASSIFICATION } \\
\text { OF ABSTRACT } \\
\text { Unclassified }\end{array}$ & 20. LIMITATION OF ABSTAACT \\
\hline
\end{tabular}

